\title{
Three-dimensional reconstruction of the intra-cluster medium
}

\author{
E. Puchwein and M. Bartelmann
}

\author{
Zentrum für Astronomie der Universität Heidelberg, ITA, Albert-Überle-Str. 2, 69120 Heidelberg, Germany \\ e-mail: puchwein@ita.uni-heidelberg.de
}

Received 19 December 2005 / Accepted 17 May 2006

ABSTRACT

\begin{abstract}
We propose and test a new method based on Richardson-Lucy deconvolution to reconstruct three-dimensional gas density and temperature distributions in galaxy clusters from combined X-ray and thermal Sunyaev-Zel'dovich observations. Clusters are assumed to be axially symmetric and arbitrarily inclined with respect to the line-of-sight. No equilibrium assumption other than local thermal equilibrium is needed. We test the algorithm with synthetic observations of analytically modeled and numerically simulated galaxy clusters and discuss the quality of the density and temperature reconstructions in idealised situations and in presence of observational noise, deviations from axial symmetry and cluster substructure. We find that analytic and numerical gas density and temperature distributions can be accurately reconstructed in three dimensions, even if observational noise is present. We also discuss methods for determining the inclination angle from data and show that it can be constrained using X-ray temperature maps. For a realistic cluster and including observational noise the three-dimensional reconstructions reach a level of accuracy of about $15 \%$.
\end{abstract}

Key words. galaxies: clusters: general - X-rays: galaxies: clusters - submillimeter

\section{Introduction}

In hierarchical models of structure formation, galaxy clusters are not only the most massive gravitationally bound objects in the Universe, but also the most recently forming. Numerous examples show that they are typically irregularly shaped and occasionally undergoing violent merger events. Cluster-sized darkmatter halos in simulations can often be well described as triaxial ellipsoids, but not as spheres (Jing \& Suto 2002).

At the same time, observations of galaxy clusters are often interpreted based on spherically-symmetric models in hydrostatic equilibrium. The beta model (Cavaliere \& Fusco-Femiano 1976) is still routinely being used for analyses of the $\mathrm{X}$-ray emission and also of the amplitude of the thermal Sunyaev-Zel'dovich effect. Given the rapidly improving quality and diversity of cluster data, it appears timely to search for an algorithm which avoids the assumption of spherical symmetry and allows the joint analysis of different types of cluster data.

Several such algorithms have been proposed. Zaroubi et al. (1998) suggested to base the reconstruction of axisymmetric, three-dimensional gravitational cluster potentials on the Fourier slice theorem, extrapolating Fourier modes into the "cone of ignorance". They applied their technique to simulated data and showed that it performs well (Zaroubi et al. 2001). Doré et al. (2001) followed a perturbative approach, and Lee \& Suto (2004) proposed to adapt parameters of triaxial halo models, all by combining different data sets such as X-ray, (thermal) Sunyaev-Zel'dovich (SZ) and gravitational-lensing maps. A similar method was applied to data by De Filippis et al. (2005). An alternative approach based on the iterative RichardsonLucy deconvolution was suggested by Reblinsky (2000) and Reblinsky \& Bartelmann (2001). It aims at the gravitational potential, assumes only axial symmetry of the main cluster body, avoids extrapolations in Fourier space, and can easily be extended to include additional data sets.
In this paper, we develop the latter algorithm further. However, aiming at the potential would require us to assume a relation between the gas distribution and the gravitational field, which would be most conveniently given by hydrostatic equilibrium. But even ignoring this common equilibrium assumption, it should be possible to reconstruct the three-dimensional distributions of intra-cluster gas density and temperature by a joint analysis of X-ray and thermal SZ data.

We demonstrate here that this is indeed possible under the one simplifying assumption that the underlying threedimensional distributions be axially (not spherically!) symmetric. The inclination of the symmetry axis can be arbitrary. We introduce the algorithm in Sect. 2 and apply it to the idealised case of an analytically modeled, ellipsoidal cluster without substructure in Sect. 3. Results obtained first without, then with observational noise are highly promising: both the three-dimensional density and temperature distributions are accurately reproduced. Noise suggests smoothing, and we describe a suitable smoothing algorithm.

We study the less-ideal case of a numerically-simulated galaxy cluster in Sect. 4. Here, axial symmetry is typically violated by the main cluster body, and substructures are present which further perturb the symmetry. Yet, faithful reconstructions are possible even in presence of realistic noise.

Section 5 finally describes how inclination angles can be constrained using temperature maps, and Sect. 6 summarises and discusses our results.

\section{The deprojection algorithm}

\subsection{Deprojection of axisymmetric quantities using Richardson-Lucy deconvolution}

As Binney et al. (1990) pointed out, Richardson-Lucy deconvolution (Lucy 1974, 1994) can be used to reconstruct an inclined axisymmetric three-dimensional distribution of some physical 


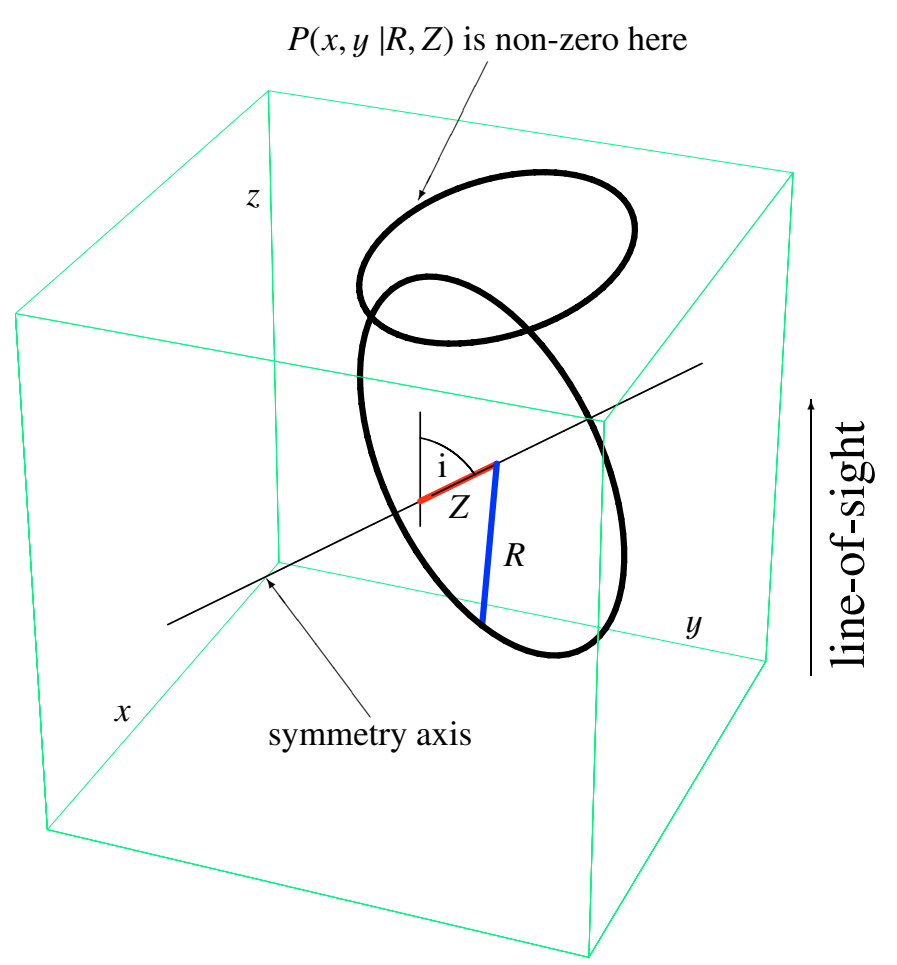

Fig. 1. Projection of an axisymmetric distribution of a physical quantity. The ellipse at the top marks the region where the kernel function corresponding to the projection along the line-of-sight is non-zero for fixed $R$ and $Z$.

quantity $\phi$ from a two-dimensional map $\psi$ of its projection along the line-of-sight. In astrophysical applications, $\psi$ will be data obtained from observations, for example the X-ray flux, the lensing potential, or the Sunyaev-Zel'dovich decrement of an approximately axisymmetric galaxy cluster. Because of the assumed symmetry, $\phi$ can be written as a function of only two cylindrical coordinates $R$ and $Z$, where we choose the symmetry axis as the $Z$-axis (see Fig. 1). Then, $R$ is the distance from the symmetry axis. The projection along the line-of-sight can be understood as a convolution of $\phi(R, Z)$ with a kernel function $P(x, y \mid R, Z)$,

$\psi(x, y) \equiv \int_{-\infty}^{\infty} \mathrm{d} z \phi(x, y, z)=\int_{0}^{\infty} \pi \mathrm{d} R^{2} \int_{-\infty}^{\infty} \mathrm{d} Z \phi(R, Z) P(x, y \mid R, Z)$.

The kernel function for a given pair $(R, Z)$ is non-zero only on the ellipse obtained by projecting the ring onto the sky which is defined by $R$ and $Z$ (see Fig. 1). It is derived in Appendix A of Binney et al. (1990) and reads

$P(x, y \mid R, Z)=\frac{\delta\left[\left(\frac{y}{\cos i}-Z \tan i\right)^{2}-\left(R^{2}-x^{2}\right)\right]}{\pi \cos i}$,

where $i$ is the inclination angle of the symmetry axis, defined as the angle between the symmetry axis and the line-of-sight; see Fig. 1. The kernel $P(x, y \mid R, Z)$ satisfies the normalisation condition

$\int_{-\infty}^{\infty} \mathrm{d} x \int_{-\infty}^{\infty} \mathrm{d} y P(x, y \mid R, Z)=1$.

Assuming that the orientation of the symmetry axis is known and that one has a two-dimensional map of the projection $\psi$, one can reverse the convolution using the iterative Richardson-Lucy deconvolution technique (see Lucy 1974, 1994) and solve for $\phi$ as a function of $R$ and $Z$. Starting with an initial guess $\phi_{0}$ for $\phi$ and using the Richardson-Lucy iteration scheme,

$\phi_{n+1}(R, Z)=\phi_{n}(R, Z) \int_{-\infty}^{\infty} \mathrm{d} x \int_{-\infty}^{\infty} \mathrm{d} y \frac{\psi(x, y)}{\psi_{n}(x, y)} P(x, y \mid R, Z)$,

one can obtain approximations of $\phi$ with increasing quality. Here, $\psi_{n}$ is the projection along the line-of-sight of the approximation $\phi_{n}$. If we plug Eq. (2) into Eq. (4), perform the $y$ integration, and use the new coordinate $\alpha$ defined by $x=R \cos \alpha$, we obtain (see also Binney et al. 1990; Reblinsky 2000)

$\frac{\phi_{n+1}(R, Z)}{\phi_{n}(R, Z)}=\int_{0}^{2 \pi} \frac{\mathrm{d} \alpha}{2 \pi} \frac{\psi(R \cos \alpha, Z \sin i+R \sin \alpha \cos i)}{\psi_{n}(R \cos \alpha, Z \sin i+R \sin \alpha \cos i)}$,

where the integration follows the ellipse shown in Fig. 1.

For numerical reconstructions of axisymmetric threedimensional distributions, we replace the integral in Eq. (5) by a sum over points, which are distributed along the ellipse and equally spaced in $\alpha$. To evaluate the sum, we need to find the ratio $\psi / \psi_{n}$ at these points. We use two grids for the iteration, one in $x, y$-space for $\psi$ and $\psi_{n}$, and one in $R, Z$-space for $\phi_{n}$. First, we project $\phi_{n}$ along the line-of-sight on the grid in $x, y$-space to find $\psi_{n}$. We do not use the kernel function for that, but perform a direct summation using a discretised version of the first equality in Eq. (1). The projection integral is approximated by a sum over $N_{z}$ equally spaced points that cover a section of length $L_{z}$ of the line-of-sight. This section is centred on the $z$-coordinate of the halo. Then, $\psi_{n}$ is obtained by

$\psi_{n}\left(x_{j}, y_{k}\right)=\frac{L_{z}}{N_{z}} \sum_{l=1}^{N_{z}} \phi_{n}\left(R\left(x_{j}, y_{k}, z_{l}\right), Z\left(x_{j}, y_{k}, z_{l}\right)\right)$

where $x_{j}$ and $y_{k}$ are the $x$ and $y$ coordinates of the grid point $(j, k)$. The $z_{l}$ are the $z$ coordinates of the $N_{z}$ points used for the projection. The function $\phi_{n}\left(R\left(x_{j}, y_{k}, z_{l}\right), Z\left(x_{j}, y_{k}, z_{l}\right)\right)$ is approximated by bilinear interpolation from the values of $\phi_{n}$ at neighbouring grid points in $R, Z$-space. Since we know $\psi$ and have calculated the projection $\psi_{n}$ of $\phi_{n}$, we can find the ratio $\psi / \psi_{n}$ at points on the ellipse by bilinear interpolation from neighbouring points of the $x, y$-space grid. This allows us to approximate the integral in Eq. (5) by a sum over $N_{\alpha}$ points,

$\frac{\phi_{n+1}(R, Z)}{\phi_{n}(R, Z)}=\frac{1}{N_{\alpha}} \sum_{m=1}^{N_{\alpha}} \frac{\psi\left(R \cos \alpha_{m}, Z \sin i+R \sin \alpha_{m} \cos i\right)}{\psi_{n}\left(R \cos \alpha_{m}, Z \sin i+R \sin \alpha_{m} \cos i\right)}$,

and find $\phi_{n+1}$ which completes the iteration step. Here $\alpha_{m}=2 \pi m / N_{\alpha}$.

\subsection{Boundary effects, artifacts and regularisation}

There is, however, a problem. Assume that $L_{z}$ corresponds to the height of the box shown in Fig. 1, and that the area covered by our map of $\psi$ corresponds to its top surface. To calculate $\psi_{n+1}$ there, we have to know $\phi_{n+1}$ everywhere in the box. But for finding $\phi_{n+1}$ close to the corners of the box, we have to evaluate Eq. (7) along ellipses that do not fit into the top surface of the box. This means that some of the points we have to sum over lie outside our map of $\psi$ and $\psi_{n}$. As suggested by Reblinsky (2000), we replace $\psi / \psi_{n}$ for those points by its value at the closest point at the perimeter of the map. This leads to some artifacts in the reconstruction of $\phi$ for large $R$ and $Z$, but yields very good results in the central region, which we are most interested in.

To start the iteration, we have to choose a guess or prior $\phi_{0}$. We adopt the simplest choice of a flat or constant prior. We set 
without regularisation

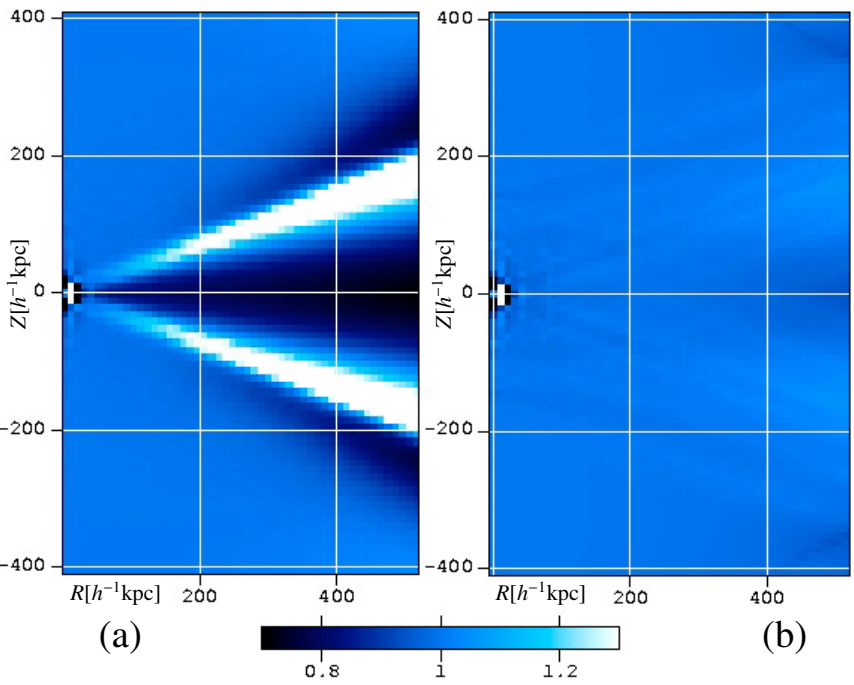

Fig. 2. Ratio of the reconstructed to the original X-ray emissivity after $n=30$ iteration steps, $\phi_{n} / \phi$. The halo centre is at the centre left of each plot. Panel a) shows the ratio obtained without regularisation. One can clearly see the spike-shaped artifacts of the reconstruction. Panel b) shows the ratio for the reconstruction including the regularisation. It is close to unity everywhere except very close to the centre, where the algorithm converges slowly and grid resolution becomes a problem.

its value so as to reproduce the average value $\langle\psi\rangle$ of the map $\psi$, namely $\phi_{0}=\langle\psi\rangle / L_{z}$.

The algorithm described above can be used to reconstruct axisymmetric three-dimensional distributions from twodimensional maps of its projection along the line-of-sight. However, it runs into problems for strongly peaked distributions such as the X-ray emissivity of a galaxy cluster. In order to illustrate that, we reconstructed the X-ray emissivity from an X-ray flux map, which we obtained by projecting the emissivity of an analytically modeled, axisymmetric cluster halo. The halo model is discussed in Sect. 3. For the projection, we chose an inclination angle of $i=70^{\circ}$. We performed the reconstruction with a rather high number of $n=30$ iterations. In the left panel of Fig. 2, we show the ratio between the reconstructed and the original X-ray emissivity. One can clearly see spike-shaped artifacts of the reconstruction. The angle between these spikes and the symmetry axis is equal to the inclination $i$. This means that the ellipses corresponding to $R$ and $Z$ values of points in the spikes pass directly through the halo centre in the map of $\psi$.

Richardson-Lucy deconvolution reproduces large structures quickly, while it converges slowly to small scale structures such as the peak at the halo centre (see Lucy 1974, 1994). This means that, when starting with a flat prior, $\psi / \psi_{n}$ can be quite large close to the centre even after several iterations. Thus, when we evaluate (7) for points further out whose ellipses pass through the halo centre, we find ratios of $\phi_{n+1}(R, Z) / \phi_{n}(R, Z)$ which are too high, and the spike-shaped artifacts form. They appear already after the first few iterations and are very stable. In the left panel of Fig. 2, we show them after 30 iterations, and it would take several hundred more iterations until they slowly disappear.

To prevent the formation of such artifacts, we use a regularisation scheme. First, we calculate an average $\left\langle\psi / \psi_{n}\right\rangle$ for the points used in the sum in Eq. (7), which is defined by

$$
\left\langle\psi / \psi_{n}\right\rangle(R, Z) \equiv \frac{1}{N_{\alpha}} \sum_{m=1}^{N_{\alpha}} \min \left(\frac{\psi\left(R \cos \alpha_{m}, Z \sin i+R \sin \alpha_{m} \cos i\right)}{\psi_{n}\left(R \cos \alpha_{m}, Z \sin i+R \sin \alpha_{m} \cos i\right)}, 10\right) .
$$

Then we set

$$
\begin{array}{r}
c_{n}(R, Z) \equiv \frac{1}{N_{\alpha}} \sum_{m=1}^{N_{\alpha}} \min \left(\frac{\psi\left(R \cos \alpha_{m}, Z \sin i+R \sin \alpha_{m} \cos i\right)}{\psi_{n}\left(R \cos \alpha_{m}, Z \sin i+R \sin \alpha_{m} \cos i\right)},\right. \\
\left.1.2\left\langle\psi / \psi_{n}\right\rangle(R, Z)\right),
\end{array}
$$

and use

$$
\frac{\phi_{n+1}(R, Z)}{\phi_{n}(R, Z)}=\max \left(c_{n}(R, Z), 0.25\right)
$$

to calculate $\phi_{n+1}(R, Z)$. This regularisation of the iteration scheme supresses the formation of the spike-shaped artifacts. It limits the impact of sharp peaks in $\psi$ on points that are far away from the corresponding peaks in $\phi$ by using an upper limit for $\psi / \psi_{n}$ in Eqs. (8) and (9). The effectiveness of the regularisation scheme is not very sensitive to the exact numerical values of the upper limits, which we chose by trial and error, as long as they suppress sharp peaks and are not too restrictive to allow convergence in a reasonable number of iterations. The lower limit for the correction factor in Eq. (10) is introduced just to make sure that $\phi_{n+1}$ does not change its sign or become very small in the first few iteration steps, which would potentially cause problems later.

We repeated the reconstruction of the X-ray emissivity using this regularisation. In the right panel of Fig. 2, we show the ratio of the reconstructed to the original emissivity after 30 iterations. The spikes that are present in the left panel have almost disappeared. The ratio is close to unity everywhere in the region shown, except very near the halo centre where grid resolution and the slow convergence to small-scale structures becomes a problem. Apart from that, the deprojection works very well. The errors are usually smaller than $1 \%$.

\subsection{Inclination angle}

So far, we have assumed that the orientation of the symmetry axis is known. In reality, when applying this algorithm to observations, this will not be the case. However, the orientation of the symmetry axis in the plane of the sky can directly be obtained from the map $\psi$. Its inclination $i$ on the other hand can be found in the following way. First, we repeat the reconstruction as described above, assuming several different plausible values of $i$ and using a fixed number $N_{\text {it }}$ of iterations. Then, we compare the maps $\psi_{N_{\mathrm{it}}}$, corresponding to the reconstructed distributions, to the original (or observed) map $\psi$ and find the value of $i$ for which it fits best. This can for example be done by minimising the penalty function

$$
\sum_{j, k} \frac{\left(\psi\left(x_{j}, y_{k}\right)-\psi_{N_{\mathrm{it}}}\left(x_{j}, y_{k}\right)\right)^{2}}{\psi^{2}\left(x_{j}, y_{k}\right)}
$$

where, depending on the shape of the distribution one wants to reconstruct, it may be favourable to sum only over points in the central region of the map. 


\subsection{Reconstruction of density and temperature of the ICM from combined $X$-ray and thermal Sunyaev-Zel'dovich effect observations}

So far, we have discussed how to reconstruct a single threedimensional distribution of a physical quantity from a single two-dimensional map of its projection along the line-of-sight. However, one can obtain additional information by combining maps from different observations (see also Reblinsky \& Bartelmann 2001; Reblinsky 2000). Here, we reconstruct several physical quantities at the same time by combining different observations that depend on these quantities. Specifically, we will show how to obtain three-dimensional distributions of the density and temperature of the intra-cluster medium (ICM) in axisymmetric cluster halos by combining observations of the (thermal) X-ray emission and the (thermal) Sunyaev-Zel'dovich effect. The X-ray surface brightness is taken to be proportional to

$\psi_{\mathrm{X}-\text { ray }} \equiv \int \mathrm{d} z \rho^{2} \lambda(T, \mathcal{Z})$,

where $\rho$ and $T$ are the gas density and temperature, respectively. The integral extends along the line-of-sight. The cooling function $\lambda(T, \mathcal{Z})$ depends on the temperature and the metallicity $\mathcal{Z}$, and $\mathcal{Z}$ is assumed to be constant here. The (thermal) Sunyaev-Zel'dovich decrement or increment is proportional to the Compton $y$-parameter

$y=\int \frac{k_{\mathrm{B}} T}{m_{\mathrm{e}} c^{2}} n_{\mathrm{e}} \sigma_{\mathrm{T}} c \mathrm{~d} t$,

where the integration also follows the line-of-sight, $k_{\mathrm{B}}$ is Boltzmann's constant, $m_{\mathrm{e}}$ the electron mass, $c$ the speed of light, $\sigma_{T}$ the Thomson cross section, and $n_{\mathrm{e}}$ the electron number density at the position passed by the light ray at time $t$. For fully ionised gas with constant metallicity, this is also proportional to $\psi_{\text {SZ }}$ defined by

$\psi_{\mathrm{SZ}} \equiv \int \mathrm{d} z \rho T$.

$\psi_{\mathrm{X} \text {-ray }}$ and $\psi_{\mathrm{SZ}}$ can be obtained from observations. For reconstructing the ICM temperature and density, we start from some initial guess $\rho_{0}(R, Z)$ and $T_{0}(R, Z)$. In analogy to Eq. (6), we use discrete approximations of Eqs. (12) and (14),

$$
\begin{aligned}
\psi_{\mathrm{X}-\mathrm{ray}, n}\left(x_{j}, y_{k}\right)= & \frac{L_{z}}{N_{z}} \sum_{l=1}^{N_{z}} \rho_{n}^{2}\left(R\left(x_{j}, y_{k}, z_{l}\right), Z\left(x_{j}, y_{k}, z_{l}\right)\right) \\
\times & \lambda\left(T\left(R\left(x_{j}, y_{k}, z_{l}\right), Z\left(x_{j}, y_{k}, z_{l}\right)\right), \mathcal{Z}\right), \\
\psi_{\mathrm{SZ}, n}\left(x_{j}, y_{k}\right)= & \frac{L_{z}}{N_{z}} \sum_{l=1}^{N_{z}} \rho_{n}\left(R\left(x_{j}, y_{k}, z_{l}\right), Z\left(x_{j}, y_{k}, z_{l}\right)\right) \\
& \times T\left(R\left(x_{j}, y_{k}, z_{l}\right), Z\left(x_{j}, y_{k}, z_{l}\right)\right),
\end{aligned}
$$

to obtain $\psi_{\mathrm{X} \text {-ray, } 0}$ and $\psi_{\mathrm{SZ}, 0}$. In analogy to Eq. (7), we define

$$
\begin{aligned}
& c_{\mathrm{X}-\mathrm{ray}, n}(R, Z)= \\
& \frac{1}{N_{\alpha}} \sum_{m=1}^{N_{\alpha}} \frac{\psi_{\mathrm{X}-\mathrm{ray}}\left(R \cos \alpha_{m}, Z \sin i+R \sin \alpha_{m} \cos i\right)}{\psi_{\mathrm{X} \text {-ray }, n}\left(R \cos \alpha_{m}, Z \sin i+R \sin \alpha_{m} \cos i\right)}, \\
& c_{\mathrm{SZ}, n}(R, Z)= \\
& \frac{1}{N_{\alpha}} \sum_{m=1}^{N_{\alpha}} \frac{\psi_{\mathrm{SZ}}\left(R \cos \alpha_{m}, Z \sin i+R \sin \alpha_{m} \cos i\right)}{\psi_{\mathrm{SZ}, n}\left(R \cos \alpha_{m}, Z \sin i+R \sin \alpha_{m} \cos i\right)},
\end{aligned}
$$

and use the iteration scheme

$$
\begin{aligned}
& \frac{\rho_{n+1}^{2} \lambda\left(T_{n+1}, \mathcal{Z}\right)}{\rho_{n}^{2} \lambda\left(T_{n}, \mathcal{Z}\right)}=c_{\mathrm{X}-\mathrm{ray}, n}, \\
& \frac{\rho_{n+1} T_{n+1}}{\rho_{n} T_{n}}=c_{\mathrm{SZ}, n} .
\end{aligned}
$$

In order to find the next iterative approximation for the density and the temperature, we have to solve Eqs. (19) and (20) for $\rho_{n+1}$ and $T_{n+1}$. To include line emission in the cooling function, one could tabulate $\lambda\left(T_{n}, \mathcal{Z}\right)$, e.g. using the software package XSPEC (see e.g. Arnaud 1996) for a specific emission model and metallicity, and solve the equations above numerically. However, since the main focus of this paper is to demonstrate the deprojection algorithm, we use the simple assumption of continuous bremsstrahlung,

$\lambda(T, \mathcal{Z})=\sqrt{T}$.

Then, we obtain from (19) and (20),

$\rho_{n+1}=\frac{c_{\mathrm{X}-\text { ray }, n}^{2 / 3}}{c_{\mathrm{SZ}, n}^{1 / 3}} \rho_{n}$
$T_{n+1}=\frac{c_{\mathrm{SZ}, n}^{4 / 3}}{c_{\mathrm{X} \text {-ray }, n}^{2 / 3}} T_{n}$.

Note that for evaluating Eqs. (17) and (18), we also use the regularisation introduced in Sect. 2.2. Its effectiveness, even when applied to different kinds of data, depends only weakly on the exact values of the numerical constants in Eqs. (8)-(10). Thus we use these equations with the numerical values given there for both the X-ray and SZ data. Again, for points that lie outside the map of $\psi_{\mathrm{X} \text {-ray }}$ and $\psi_{\mathrm{SZ}}$, the ratios $\psi_{\mathrm{X}-\text { ray }} / \psi_{\mathrm{X}-\text { ray }, n}$ and $\psi_{\mathrm{SZ}} / \psi_{\mathrm{SZ}, n}$ are approximated by their values at the closest point at the perimeter of the map. In the next two sections, we shall apply this deprojection algorithm to axisymmetric analytic halos and to numerically simulated cluster halos, and discuss its performance.

\section{Deprojection of analytic halos}

We use a NFW-like gas density profile to test the deprojection algorithm for axisymmetric, analytic halos. However, for the deprojection to be non-trivial, we prefer to have ellipsoidal isodensity surfaces. We therefore assume that the density of the ICM is a function of

$r \equiv \sqrt{\frac{R^{2}}{R_{\mathrm{s}}^{2}}+\frac{Z^{2}}{Z_{\mathrm{s}}^{2}}}$,

where $R_{\mathrm{S}}$ is a scaling radius perpendicular to the symmetry axis, and $Z_{\mathrm{s}}$ is a scaling distance along the axis. The density of the hot cluster gas is then taken to be

$\rho=\frac{\rho_{\mathrm{s}}}{\left(\epsilon_{r}+r\right)(1+r)^{2}}$,

which differs from the NFW form not only by its ellipsoidal shape but also by the small constant $\epsilon_{r}=0.001$ introduced to ensure that the density does not diverge for $r \rightarrow 0$. This divergence would cause problems in numerical calculations. For the gas temperature we use a phenomenological description that roughly corresponds to the temperature profiles found in the simulated cluster sample that we will use in Sect. 4. Namely, we set $T=T_{\max } r^{-0.2 \gamma(r)}$,

where $\gamma(r)=\tanh (3(r-1))$ is -1 for $r \ll 1$ and +1 for $r \gg 1$. The values of the parameters $\rho_{\mathrm{s}}=3 \times 10^{5} h^{-1} M_{\odot} /\left(h^{-1} \mathrm{kpc}\right)^{3}$, $Z_{\mathrm{s}}=500 h^{-1} \mathrm{kpc}, R_{\mathrm{s}}=300 h^{-1} \mathrm{kpc}$, and $T_{\max }=12 \mathrm{keV}$, which we use correspond to the gas component of a massive galaxy cluster. Here, $h$ is the reduced Hubble constant, which we set to 0.7 . 


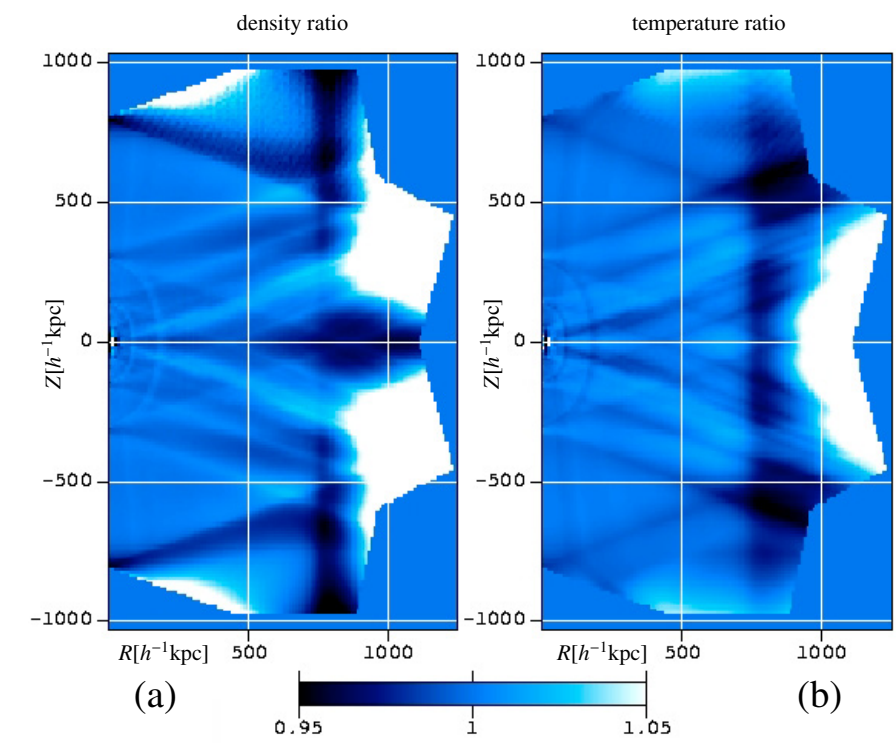

Fig. 3. Ratio of the reconstructed to the original density and temperature for the analytic halo after $n=20$ iterations. An inclination angle of $i=70^{\circ}$ was chosen and assumed to be known in performing the reconstruction. Panel a) shows the density ratio $\rho_{n} / \rho$ and panel b) the temperature ratio $T_{n} / T$. In the central region, the errors are of the order of $1 \%$. We plot the ratios for all $R$ and $Z$ values possible within the box used for the reconstruction (see Fig. 1). This causes the star-like shape of the perimeter of the plot in the $R, Z$ plane. Close to that boundary, at large $R$ or $Z$ values, the ratios can significantly differ from unity. This is, however, expected because the ellipses used in the reconstruction of $\rho$ and $T$ at those points lie mostly outside the maps of $\psi_{\mathrm{X} \text {-ray }}$ and $\psi_{\mathrm{SZ}}$.

\subsection{Deprojection of analytic halos without observational noise}

Having chosen an inclination angle $i$, we project the analytic halo described above on a $128 \times 128$ grid with a sidelength of $1.5 h^{-1} \mathrm{Mpc}$ and obtain the X-ray and Sunyaev-Zel'dovich effect maps, $\psi_{\mathrm{X} \text {-ray }}$ and $\psi_{\mathrm{sz}}$. We use the algorithm discussed in Sect. 2.4 with these maps to reconstruct the gas density and temperature. In Fig. 3, we compare the results of the deprojection with the original density and temperature of the analytic halo for an inclination angle $i=70^{\circ}$ and after $n=20$ iterations. The inclination was assumed to be known in performing the deprojection. The left and right panels show the density and temperature ratios, $\rho_{n} / \rho$ and $T_{n} / T$, repsectively. The star-like pattern of the plots maps the ranges of the $R$ and $Z$ coordinates occurring in the simulation box used for the reconstruction (see Fig. 1). In the central region of the cluster, the reconstruction works very well. Errors are of the order of 1\%. Despite the regularisation, one can still see some remains of the spike-shaped artifacts discussed in Sect. 2.2. For large $R$ or $Z$ values, close to the star-shaped boundary of the plots, the quality of the reconstruction decreases. This is not at all surprising because the ellipses along which Eqs. (17) and (18) must be evaluated to reconstruct density and temperature at those points lie mostly outside of the maps of $\psi_{\mathrm{X} \text {-ray }}$ and $\psi_{\text {sz. }}$.

Note that the quality of the reconstruction also depends on the inclination of the halo's symmetry axis. Of course, best results are achieved when the symmetry axis is perpendicular to the line-of-sight. Then the assumption of axial symmetry contains the most information. If, on the other hand, the symmetry axis is parallel to the line-of-sight, the axial symmetry just corresponds to the circular symmetry of the maps $\psi_{\mathrm{X}-\text { ray }}$ and $\psi_{\mathrm{SZ}}$ and

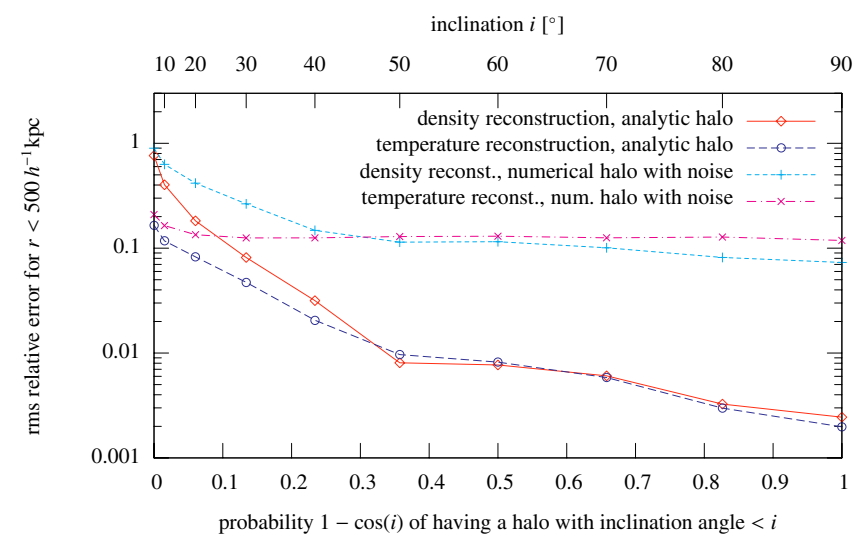

Fig. 4. Dependence of the quality of the deprojection on the inclination of the symmetry axis. For the analytic halo, which was deprojected as described above, we show the volume-weighted rms relative errors $\left(\rho_{n}-\rho\right) / \rho$ and $\left(T_{n}-T\right) / T$ as functions of the inclination angle $i$ after $n=20$ iterations. We also show the same errors after $n=5$ iterations for reconstructions of a numerical halo from maps with observational noise. A detailed description of these reconstructions is given in Sect. 4. The averages of the errors were computed within a sphere of radius $500 h^{-1} \mathrm{kpc}$ around the halo centre. The quantity $1-\cos (i)$ shown on the abscissae is chosen such as to have a flat number-density distribution for randomly oriented halos. The inclination angle $i$ was assumed to be known in performing the deprojection.

does not yield any useful additional information. Figure 4 illustrates this inclination dependence. It shows the volume-weighted root mean square (rms) relative errors of the reconstructed gas density and temperature, computed within a sphere of radius $500 h^{-1} \mathrm{kpc}$ around the halo centre. Again, the knowledge of the inclination angle $i$ was used in the deprojection. An accuracy of $1 \%$ or better is achieved for about two thirds of the analytic halos in a randomly oriented sample. However, halos that happen to have a very small inclination angle $i$ are necessarily poorly reconstructed.

\subsection{Deprojection of analytic halos including observational noise}

So far we have not considered noise that will be present in any real X-ray or Sunyaev-Zel'dovich effect observation. We will now discuss the impact it has on the reconstruction of ICM densities and temperatures.

We model the noise in X-ray observations as follows. First, we calculate for each pixel $(j, k)$ of the halo's X-ray map $\psi_{\mathrm{X} \text {-ray }}$ the number of photons $\left\langle N_{\gamma j, k}\right\rangle$ expected from bremsstrahlung, which is proportional to $\left\langle N_{\gamma j, k}\right\rangle \sim \sum_{l=1}^{N_{Z}} \mathrm{E}_{1}\left(\frac{E_{\min }}{k_{B} T}\right) \frac{\rho^{2}}{\sqrt{T}}$, where the sum extends along the line-of-sight represented by the pixel $(j, k)$, and $\mathrm{E}_{1}$ is the exponential integral function. $E_{\min }$ is a lower energy cutoff which is necessary because the number of photons emitted is infrared divergent. We choose $E_{\min }=0.23 \mathrm{keV}$, which is a reasonable lower limit for the photons from galaxy clusters observed in current X-ray experiments. Next, we normalise the numbers of expected photons such that they sum up to $\sum_{j, k}\left\langle N_{\gamma j, k}\right\rangle=10^{4}$ on the entire map. For each pixel $(j, k)$, we then set the actual number of photon counts $N_{\gamma j, k}$ to a value drawn from a Poisson distribution with expectation value $\left\langle N_{\gamma j, k}\right\rangle$. We then add the noise to the map $\psi_{\mathrm{X} \text {-ray }}$ by multiplying $\psi_{\mathrm{X} \text {-ray } j, k}$ with $N_{\gamma j, k} /\left\langle N_{\gamma j, k}\right\rangle$ for all pixels. 
For the Sunyaev-Zel'dovich effect, we add noise corresponding to future ALMA Band 3 observations (see Butler \& Wootten 1999). In Band 3 ( 84 to $116 \mathrm{GHz}$ ) and in its compact configuration, ALMA will be able to achieve a temperature sensitivity of $50 \mu \mathrm{K}$ at a spatial resolution of $\sim 3$ arcsec in about four hours of observation. At an assumed halo redshift of 0.3 , this resolution corresponds to the angular size chosen for the pixels of the map $\psi_{\text {SZ }}$. We convert the temperature sensitivity cited above to an error $\sigma_{\psi_{\mathrm{sz}}}$ of $\psi_{\mathrm{sz}}$. Then, for each pixel, we add noise obtained from a normal distribution with standard deviation $\sigma_{\psi_{\mathrm{sZ}}}$ to $\psi_{\mathrm{SZ}}$.

Richardson-Lucy deconvolution has the nice property of approximating large-scale features quickly and small-scale noise slowly. Yet, it turns out that smoothing the noisy maps $\psi_{\mathrm{X} \text {-ray }}$ and $\psi_{\mathrm{SZ}}$ before using them in the deprojection improves the results considerably. We use the following smoothing scheme. For the X-ray observations, we assume that in addition to the map $\psi_{\text {X-ray }}$ we also know the photon counts $N_{\gamma j, k}$ for all pixels. We then calculate for each pixel $(j, k)$ a radius $h_{\mathrm{SML} j, k}$ so that we have a fixed number of 100 photons inside a circle with radius $h_{\mathrm{SML} j, k}$ around that pixel. After that we redistribute the value $\psi_{\mathrm{X}-\text { ray } j, k}$ of each pixel on the grid with a smoothing kernel of width $h_{\mathrm{SML} j, k}$ centred on that pixel. This greatly reduces the fluctuations in the map $\psi_{\mathrm{X} \text {-ray }}$ caused by photon noise. In the remainder of the paper we will call this first step of the smoothing scheme "photonnoise smoothing".

For the smoothing kernel, we take the line-of-sight projection of the cubic spline SPH kernel $W\left(r, h_{\mathrm{SML}}\right)$ defined in Appendix A of Springel et al. (2001). It is well suited for this purpose and allows us to use the same routine for smoothing here and for projecting the numerical SPH halos we use in Sect. 4. For axisymmetric halos, the projection should be symmetric about the projected axis. However, the symmetry is broken here by noise. We restore it before performing the deprojection. Since we have oriented the grid such that it is parallel to and centred on the projected symmetry axis, we can do that by replacing $\psi_{\mathrm{X}-\text { ray } j, k}$ and $\psi_{\mathrm{X}-\text { ray }} N_{\text {grid }}-j, k$ by their arithmetic mean. Here, $N_{\text {grid }}=128$ is the dimension of the grid. We symmetrise $\psi_{\mathrm{SZ}}$ in the same way. After that we perform one more smoothing operation on $\psi_{\mathrm{X} \text {-ray }}$ and $\psi_{\text {SZ }}$ to further reduce fluctuations caused by noise.

In numerically simulated halos, which we will discuss later, this will also suppress the effect of subclumps. Since we do not want to smooth out the peaks in the halo core, we choose a smoothing length $h_{\mathrm{SML}}$ that depends on the distance $r$ from the halo (or map) centre, namely $h_{\mathrm{SML}}=h_{\mathrm{SML}, \max }(1-$ $\left.W\left(r, r_{\max }\right) / W\left(0, r_{\max }\right)\right)$. It is zero in the centre of the map and continually increases to $h_{\mathrm{SML}}=375 h^{-1} \mathrm{kpc}$ at a radius equal to $r_{\max }=750 h^{-1} \mathrm{kpc}$ or larger. This yields smallest rms errors. Once we have calculated $h_{\mathrm{SML}}$ for each pixel, we smooth the maps of $\psi_{\mathrm{X} \text {-ray }}$ and $\psi_{\mathrm{SZ}}$ with the projection of the SPH smoothing kernel mentioned above and with the position-dependent smoothing length $h_{\mathrm{SML}}$. Note that roughly $80 \%$ of a pixel's value is redistributed within a circle of radius $h_{\mathrm{SML}} / 2$. We refer to this second step of the smoothing scheme as "radius-dependent smoothing".

After degrading the analytic halo with noise and applying the smoothing scheme described above, we perform the iterative deprojection. The results after $n=5$ iterations are shown in Fig. 5. An inclination of $i=70^{\circ}$ was chosen and assumed to be known in the deprojection. Again, the left panel shows the ratio of the reconstructed to the original density, and the right panel the corresponding temperature ratio. Average errors in the central region are of the order of $5 \%$ to $10 \%$. As expected, further outside, where the signal-to-noise ratio becomes small and the ellipses used for the reconstruction lie mostly outside the maps

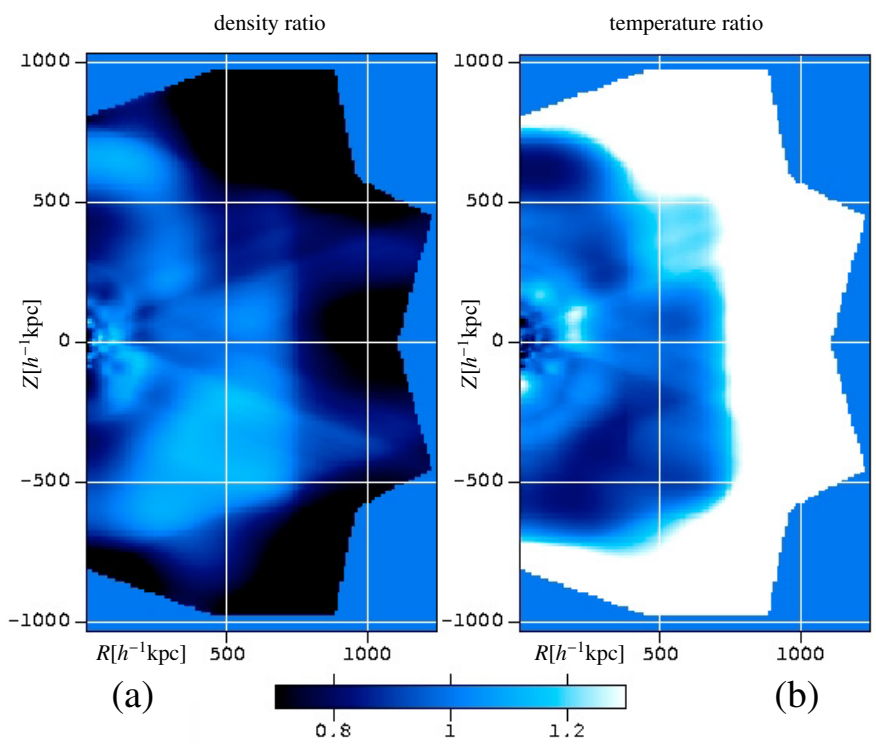

Fig. 5. Ratio of the reconstructed to the original density and temperature for the analytic halo with observational noise after $n=5$ iterations. An inclination of $i=70^{\circ}$ was chosen and assumed to be known in performing the reconstruction. "Photon-noise" and "radius-dependent" smoothing were applied. Panel a) shows the density ratio $\rho_{n} / \rho$, and panel b) the temperature ratio $T_{n} / T$. In the central region, the errors are of the order of $5 \%$ to $10 \%$.

of $\psi_{\mathrm{X}-\text { ray }}$ and $\psi_{\mathrm{SZ}}$, the errors are substantially larger. Note that at locations where we obtain a too low density, we usually find a too high temperature and vice versa. This happens because the algorithm minimises the deviations of the reconstructed from the original X-ray and SZ-effect maps.

In Fig. 6, we show density and temperature profiles of the original analytic halo, of the halo reconstructed from maps without observational noise, and of the halo reconstructed from smoothed maps which contain observational noise. The reconstructed halos are the same as shown in Figs. 3 and 5. Without noise, both the temperature and the density profiles are reproduced very well. With noise, we can still reproduce density profiles with an accuracy of a few percent. The errors in the temperature profile are somewhat larger. Deviations are mainly caused by the noise, but some are also artifacts of the smoothing scheme we apply. Especially the too high temperature near $\sim 200 h^{-1} \mathrm{kpc}$ and the too low temperature near $\sim 400 h^{-1} \mathrm{kpc}$ are a consequence of "radius-dependent smoothing". On the other hand, without such smoothing we would approximately double the errors in the density and temperature reconstructions.

\section{Deprojection of numerically simulated cluster halos}

So far, we have demonstrated the performance of the algorithm with axisymmetric analytic halos. We were able to reconstruct their three-dimensional density and temperature distributions from synthetic X-ray and (thermal) Sunyaev-Zel'dovich effect observations. Real galaxy clusters, however, are hardly perfectly axisymmetric. We will study in this section whether they nonetheless allow accurate density and temperature reconstructions with the deprojection algorithm proposed in Sect. 2.4. We use a sample of four numerically simulated galaxy clusters to investigate into this question. 


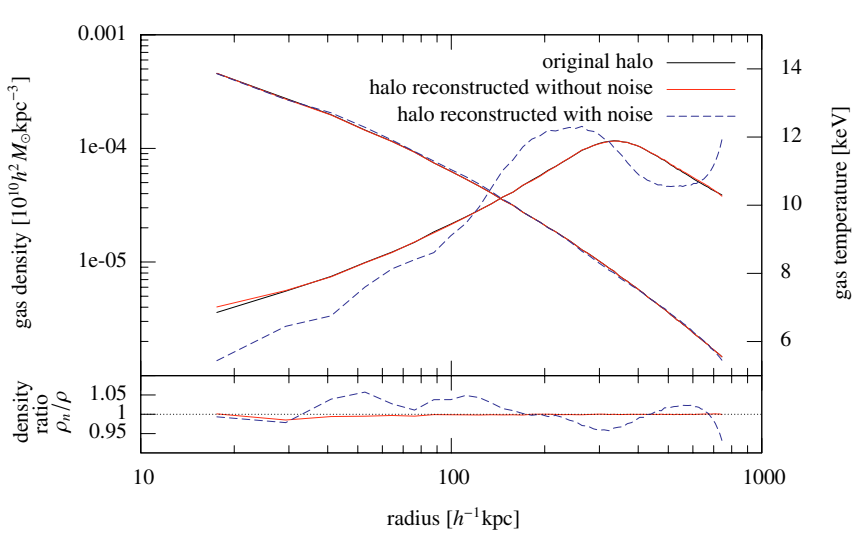

Fig. 6. Density and temperature profiles of the original and the reconstructed analytic halos. The upper panel shows the density (falling curves, left axis) and the temperature profiles (rising curves, right axis) of the original analytic halo, the halo reconstructed without observational noise (and without any smoothing), and the halo reconstructed from maps with observational noise to which the complete smoothing scheme was applied. The lower panel shows the profile of the ratio of the reconstructed density $\rho_{n}$ to the original density $\rho$. The number of iterations used was $n=20$ in the case without noise and $n=5$ in the case with noise. An inclination of $i=70^{\circ}$ was chosen and assumed to be known in the reconstruction.

The simulations were carried out by Klaus Dolag with GADGET-2 (Springel 2005), a new version of the parallel TreeSPH simulation code GADGET (Springel et al. 2001). The cluster regions were extracted from a dissipation-less (dark matter only) simulation with a box size of $479 h^{-1} \mathrm{Mpc}$ of a flat $\Lambda \mathrm{CDM}$ model with $\Omega_{\mathrm{m}}=0.3, h=0.7, \sigma_{8}=0.9$ (see Yoshida et al. 2001). They were re-simulated with higher resolution using the "Zoomed Initial Conditions" (ZIC) technique (Tormen 1997). Gas was introduced into the high-resolution region by splitting each parent particle into a gas and a dark matter particle, which were then displaced by half the mean inter-particle distance, such that the centre-of-mass and the momentum were conserved. The mass ratio of gas to dark matter particles was set to obtain $\Omega_{\mathrm{b}}=0.04$. The final mass resolution was $m_{\mathrm{DM}}=$ $1.13 \times 10^{9} h^{-1} M_{\odot}$ and $m_{\text {gas }}=1.7 \times 10^{8} h^{-1} M_{\odot}$ for dark-matter and gas particles within the high-resolution region, respectively. The simulations we use follow the dynamics of the dark matter and the adiabatic evolution of the cluster gas, but they ignore radiative cooling. They are described in more detail in Puchwein et al. (2005) and Dolag et al. (2005).

Our deprojection algorithm requires a symmetry axis, which real clusters do not generally have. We thus need to choose an axis around which the numerical clusters have at least a high degree of symmetry. We do this by calculating the inertial tensor of the cluster gas inside a sphere of radius $500 \mathrm{~h}^{-1} \mathrm{kpc}$ around the cluster centre and finding its eigenvectors $\boldsymbol{v}_{\mathbf{1}}, \boldsymbol{v}_{\mathbf{2}}, \boldsymbol{v}_{\mathbf{3}}$ and eigenvalues $e_{1} \geq e_{2} \geq e_{3}$. We choose the symmetry axis through the cluster centre and parallel to the eigenvector $\boldsymbol{v}_{3}$ with the smallest eigenvalue if $e_{1} / e_{2} \leq e_{2} / e_{3}$, or parallel to the eigenvector $v_{1}$ with the largest eigenvalue otherwise. This means that, if two eigenvalues are very similar, we choose the axis parallel to the eigenvector corresponding to the third eigenvalue.

\subsection{Deprojection of numerical halos without observational noise}

Having chosen a fiducial "symmetry" axis and a lineof-sight, we can produce synthetic maps of X-ray and

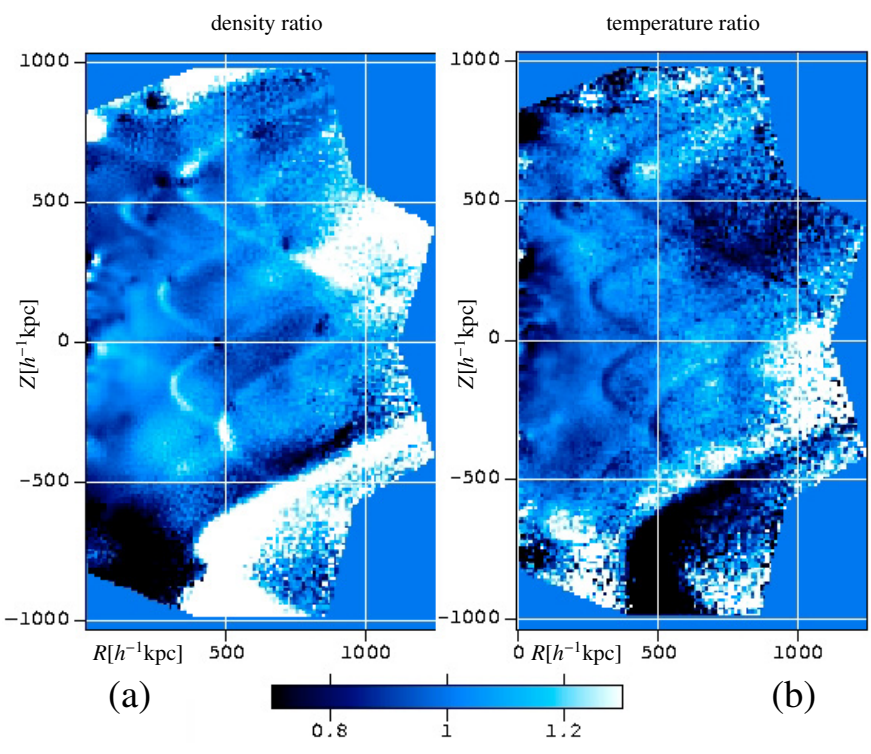

Fig. 7. Reconstruction of the simulated cluster g51 without noise and smoothing. The ratios of the reconstructed to the original density and temperature are shown. The deprojection was done with $n=5$ iterations. An inclination angle of $i=68^{\circ}$ between the line-of-sight and the principal inertial axis of the cluster gas was chosen and assumed to be known in the reconstruction. Panel a) shows the density ratio $\rho_{n} / \rho$ and panel b) the temperature ratio $T_{n} / T$. In the central region, the errors are of the order of $10 \%$.

Sunyaev-Zel'dovich effect observations. For that purpose, we use the simulated clusters at a redshift of $z=0.3$ and project them along the line-of-sight. At $z=0.3$, the cluster sample spans a mass range between $8 \times 10^{14}$ and $1.8 \times 10^{15} h^{-1} M_{\odot}$.

For now, we do not add any observational noise to the maps. However, the clusters contain substructures which break axial symmetry and lead to artifacts in the density and temperature reconstructions. Thus, depending on the amount of substructure present in a cluster, it may still be favourable to use "radiusdependent smoothing" on the X-ray and Sunyaev-Zel'dovich effect maps prior to reconstruction. In Figs. 7 and 8, we show the results of the deprojection without any smoothing, and using "radius-dependent smoothing".

The density reconstruction in the central region reaches an accuracy of about $10 \%$ in both cases. For the temperature reconstruction and the density reconstruction at large $r$, we obtain somewhat better results without smoothing for this rather symmetric cluster. Note, however, the hyperbolically shaped artifacts in Fig. 7 which are produced by substructure clumps in absence of smoothing. They appear at those $R$ and $Z$ values which correspond to the line-of-sight passing through such a clump. The spike-shaped artifacts discussed in Sect. 2.2 were a special case of the artifacts found here. For most of the hyperbolae in the left panel of Fig. 7, one can also see the position of the clump that produced it in darker colours. The hyperbolae pass right through them.

As one can see in Fig. 8, "radius-dependent smoothing" removes the hyperbolic artifacts. The subclumps, however, still appear in darker colours in the density ratio map, which means that the reconstructed density there is too low. However, this is entirely expected and inevitable, because they violate axial symmetry and thus cannot be faithfully reconstructed with this deprojection technique. By smoothing, we essentially remove the subclumps from the maps and reconstruct the density and temperature of the main halo without them. 


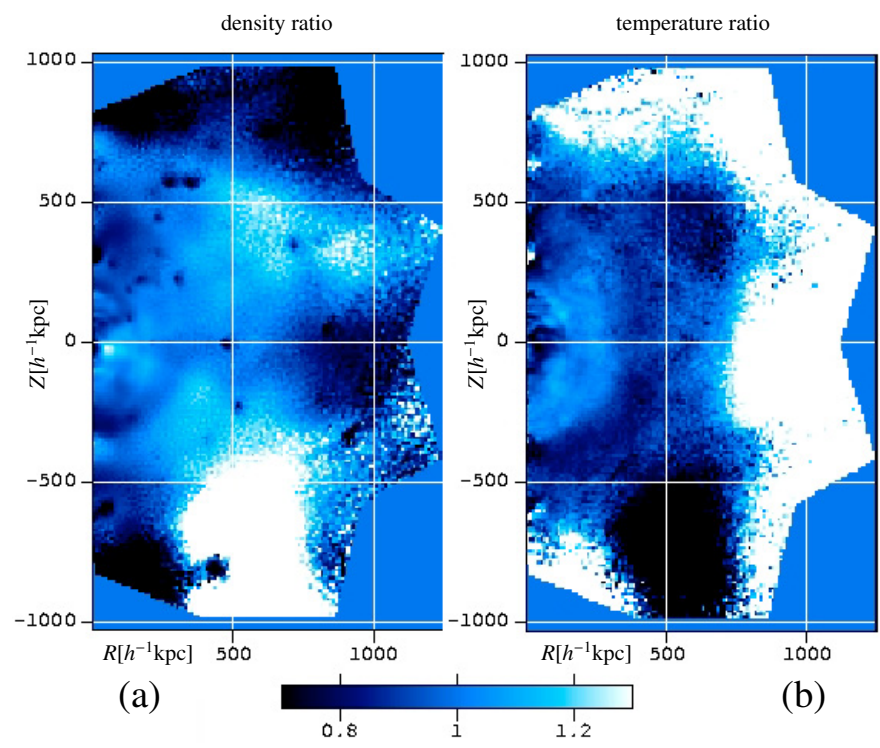

Fig. 8. Reconstruction of the simulated cluster g51 without noise but with "radius-dependent smoothing". The ratios of the reconstructed to the original density and temperature are shown. The deprojection was done with $n=5$ iterations. An inclination angle of $i=68^{\circ}$ between the line-of-sight and the principal inertial axis of the cluster gas was chosen and assumed to be known in the reconstruction. Panel a) shows the density ratio $\rho_{n} / \rho$ and panel b) the temperature ratio $T_{n} / T$. In the central region, the errors are of the order of $10 \%$.

Unfortunately, "radius-dependent smoothing" also affects the density and temperature profiles. This can be seen in the "rings" around the halo centre in the right panel of Fig. 8. It is further illustrated in Fig. 10, which shows the density and temperature profiles of the original cluster g51, after deprojection without noise but with "radius-dependent smoothing", and after deprojection without noise and without smoothing. For $r>300 h^{-1} \mathrm{kpc}$, the reconstruction without smoothing yields more accurate density and temperature profiles. In addition, the profiles for deprojections from maps including observational noise are shown. They will be discussed in the next section.

Reconstructions along different lines-of-sight and of the three other clusters in the sample gave similar results. For the most asymmetric halo, the errors were larger by factors of 1.5 to 2 compared to the reconstruction of g51 presented above. We can thus conclude that, although clusters are not strictly axisymmetric and contain substructure, it is possible to apply the deprojection method proposed in Sect. 2.4 and successfully reconstruct three-dimensional density and temperature distributions of the cluster gas.

\subsection{Deprojection of numerical halos including observational noise}

In Sect. 3.2, we studied the impact of observational noise in the X-ray and Sunyaev-Zel'dovich effect maps on the quality of the density and temperature reconstruction. We will now do the same for the numerically simulated cluster halos using the same noise model, namely Poisson noise corresponding to $10^{4}$ observed photons for the X-ray maps and a noise level expected for a four-hour ALMA Band 3 observation for the SunyaevZel'dovich effect maps. We also use the smoothing scheme described there.

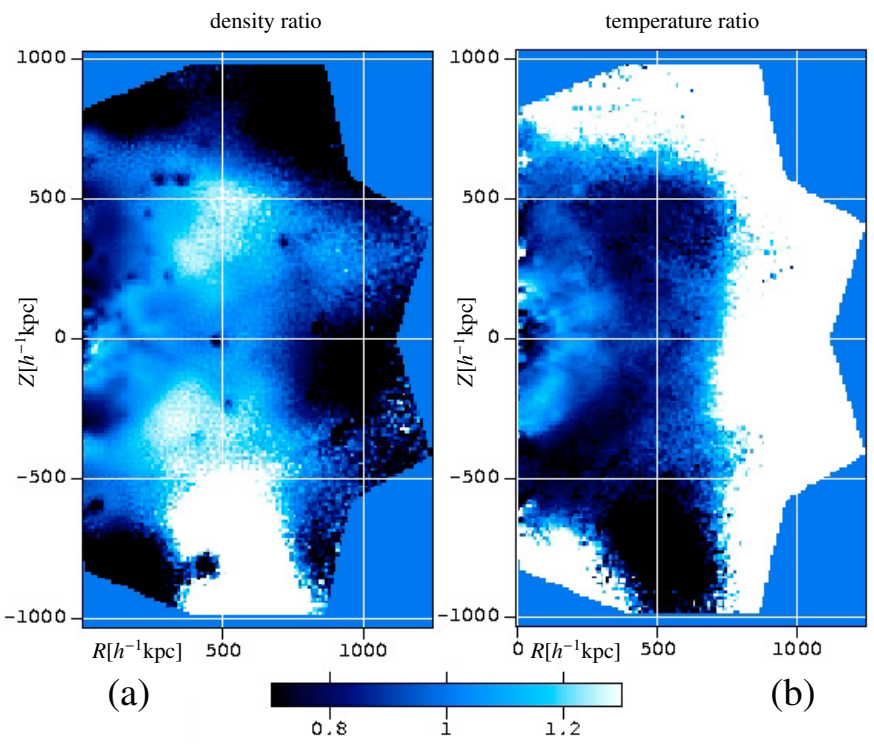

Fig. 9. Reconstruction of the simulated cluster g51 with noise and and the complete smoothing scheme applied. The ratios of the reconstructed to the original density and temperature are shown. The deprojection was done with $n=5$ iterations. An inclination angle of $i \approx 68^{\circ}$ between the line-of-sight and the principal inertial axis of the cluster gas was chosen and assumed to be known in the reconstruction. Panel a) shows the density ratio $\rho_{n} / \rho$ and panel $\left.\mathbf{b}\right)$ the temperature ratio $T_{n} / T$. In the central region, the errors are of the order of $15 \%$.

We show the results of the reconstruction in Fig. 9. Again, the left panel shows the ratio of the reconstructed to the original density, and the right panel the corresponding temperature ratio. In the central region we achieve an accuracy of about $15 \%$. Without "radius-dependent smoothing", errors would be larger by roughly a factor of 1.5 or even more next to the halo centre. However, if one is mainly interested in density and temperature profiles it may still be favourable to leave the "radius-dependent smoothing" step away. Although the errors are larger without "radius-dependent smoothing", they are less biased with respect to the distance from the halo centre and cancel better when averaging over spherical shells around it, especially at large radii. Thus depending on the quantity one is finally interested in or the context in which the reconstructions are used, different amounts of smoothing may yield best results. Figure 10 shows the profiles obtained with and without "radius-dependent smoothing".

We still need to discuss when the iteration used in the density and temperature reconstructions should best be stopped. Figure 11 illustrates the dependence of the quality of the reconstruction on the number of iterations used. More precisely, it shows the relative volume-weighted rms error of the density reconstruction within $r=500 h^{-1} \mathrm{kpc}$ as a function of the number of iterations and for different deprojection schemes, namely for the deprojections of the analytic halo and the numerically simulated cluster g51 discussed above and shown in Figs. 3, 5, 8 , and 9 after $n=20$ or $n=5$ iterations. The quality of the reconstruction improves quickly during the first roughly five iterations (first ten for the analytic halo without noise) and then levels off. In addition, we show the quality of the reconstruction of g51 from maps with noise but without using "radius-dependent smoothing". In this case, small-scale noise in the maps is not sufficiently suppressed. The best reconstruction is found after five iterations. Then, the quality decreases again because the algorithm starts to approximate small-scale noise. Thus, unless a 


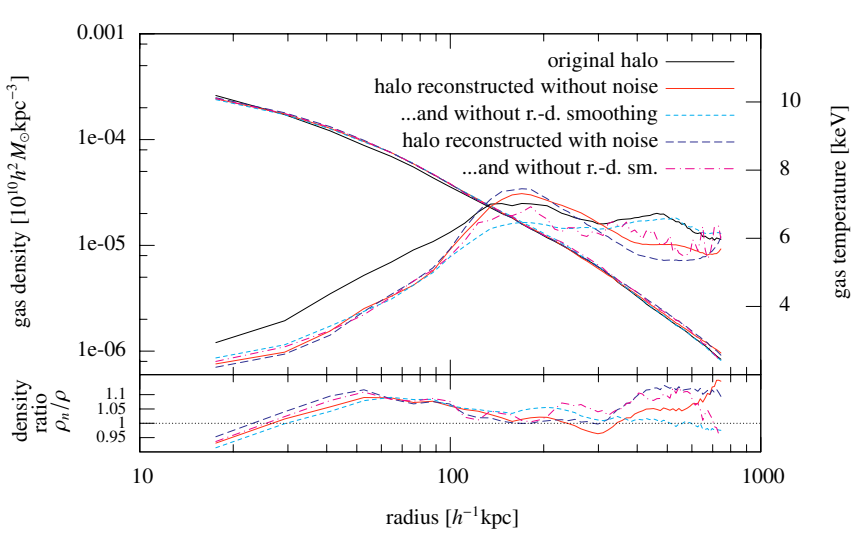

Fig. 10. Gas density and temperature profiles of the original and the reconstructed cluster g51. The upper panel shows the density profiles (falling curves, left axis) and the temperature profiles (rising curves, right axis) of the original cluster, the cluster reconstructed without observational noise but with "radius-dependent smoothing", reconstructed without observational noise and without any smoothing, reconstructed from maps with observational noise and the complete smoothing scheme applied, and reconstructed from maps with observational noise but without "radius-dependent smoothing". The lower panel shows the profile of the ratio of the reconstructed density $\rho_{n}$ to the original density $\rho$. The number of iterations used was $n=5$ in all cases. An inclination angle of $i \approx 68^{\circ}$ was chosen and assumed to be known in the reconstruction.

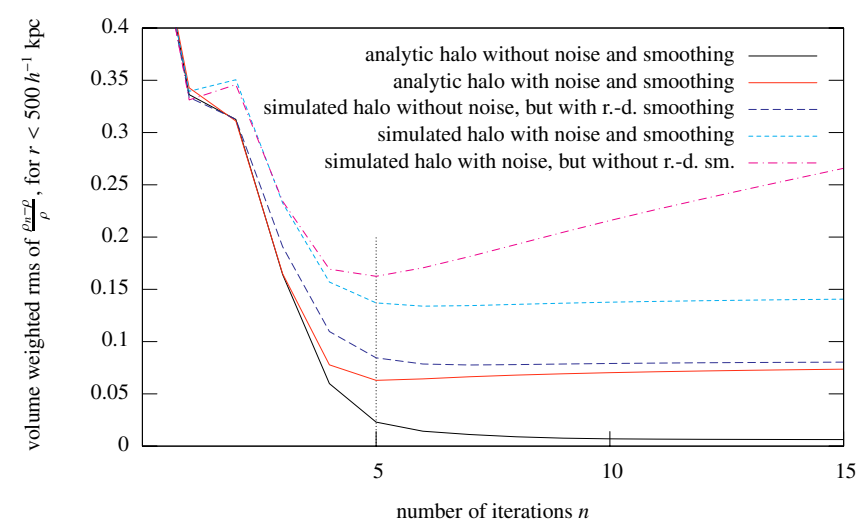

Fig. 11. Dependence of the quality of the density reconstruction on the number of iterations used. We show the volume-weighted relative rms error $\frac{\rho_{n}-\rho}{\rho}$ within spheres with $r=500 h^{-1} \mathrm{kpc}$ radius and for different deprojections of our analytic halo model and of the numerically simulated cluster g51. The figure legend explains which halo, whether or not noise, and what kind of smoothing were used for the different reconstructions shown. Note that "photon-noise smoothing" of the X-ray maps is always and only used for maps with noise.

halo is very smooth and axisymmetric, such as the analytic halo without noise, we find that the quality of the reconstruction does not significantly increase after $n=5$ iterations and may even decrease if small scale fluctuations due to noise are not efficiently suppressed. Thus, we conclude that it is favourable to use this number of iterations for the deprojection of simulated and real galaxy clusters. Alternatively, one could control the reproduction of small-scale fluctuations with a formal regularisation scheme, such as provided by maximum-entropy methods.

\section{Finding inclination angles}

In all deprojections of analytic and numerical clusters presented above, we have assumed that the inclination angle of the "symmetry" axis is known beforehand. This will usually not be the case for real observed clusters. In Sect. 2.3, we sketched how one can obtain inclination angles by comparing the maps obtained by projecting reconstructed halos to the original maps. One would reconstruct a cluster assuming different values for $i$ and find the value for which the maps match best. In principle, we could generalise this approach to the X-ray and SunyaevZel'dovich effect maps used for the density and temperature deprojections. That is, we can compare the original maps $\psi_{\mathrm{X} \text {-ray }}$ and $\psi_{\mathrm{SZ}}$ to the maps $\psi_{\mathrm{X}-\text { ray, } N_{\mathrm{it}}}$ and $\psi_{\mathrm{SZ}, N_{\mathrm{it}}}$, which correspond to the reconstructed halo after a fixed number $N_{\text {it }}$ of iterations and for different values of the inclination angle $i$ used for the reconstruction.

We did this for the analytic halo model and for our sample of numerical clusters and used different inclination angles $i^{\prime}$ for projecting these halos to obtain the original maps. However, the minima in the penalty function (see Eq. (11)) are not well defined. They are very broad and not always centred on $i=i^{\prime}$. Even for the analytic halo without observational noise, it is hardly possible to find the correct axis inclination in this way. As one can see from Eqs. (17) and (18), the iterative corrections of the deprojection algorithm are determined from the deviations of the $\mathrm{X}$-ray and Sunyaev-Zel'dovich effect maps, and the deviations are thereby minimised. Unfortunately, this still works remarkably well when choosing a wrong inclination angle $i \neq i^{\prime}$ for the deprojection. Thus the X-ray and Sunyaev-Zel'dovich effect maps are still reproduced well in this case, although the errors of the density and temperature reconstructions increase significantly.

We tried to limit the ability of the deprojection algorithm to reproduce maps well even when the inclination angle is wrong by reducing its degrees of freedom. For doing so, we used a variant of the algorithm that only reconstructs the density and uses a constant but adjustable temperature. This of course also limits the accuracy of the reconstruction for the correct inclination angle. Thus, the results of comparing the reconstructed X-ray and Sunyaev-Zel'dovich effect maps to the original ones for finding the inclination angle were not significantly better.

On the other hand, leaving the deprojection algorithm as described in Sect. 2.4, but using additional independent information for finding the inclination angle of the halo, seems to be more promising. For the deprojection, we use maps of the X-ray flux of the clusters, but so far we do not use any spectral information from the X-ray observations. In Figs. 12 and 13, we assume that in addition to the X-ray flux maps we also have maps of the emission-weighted temperature $T_{\text {ew }}$. We reconstruct the analytic halo and the numerically simulated cluster g51 from X-ray flux and Sunyaev-Zel'dovich effect maps as above, but then compare the original emission-weighted temperature map to one we obtain by projecting the reconstructed halos. We repeated this for different inclination angles $i^{\prime}$, chosen for projecting the original maps, and $i$, chosen in the reconstruction.

Note, however, when applying this method to real galaxy clusters and projected temperature maps obtained by X-ray spectral fitting of single temperature emission models to observations, it may be favourable to use a more sophisticated projected temperature definition, such as the spectroscopic-like temperature, instead of the emission-weighted temperature (see Mazzotta et al. 2004).

Figure 12 shows the rms relative error of the reconstructed emission-weighted temperature maps for the analytic halo without and with noise. $N_{\text {it }}=20$ and no smoothing were used without noise, and $N_{\text {it }}=5$ and the complete smoothing scheme were used with noise. The rms error was computed within a radius of 


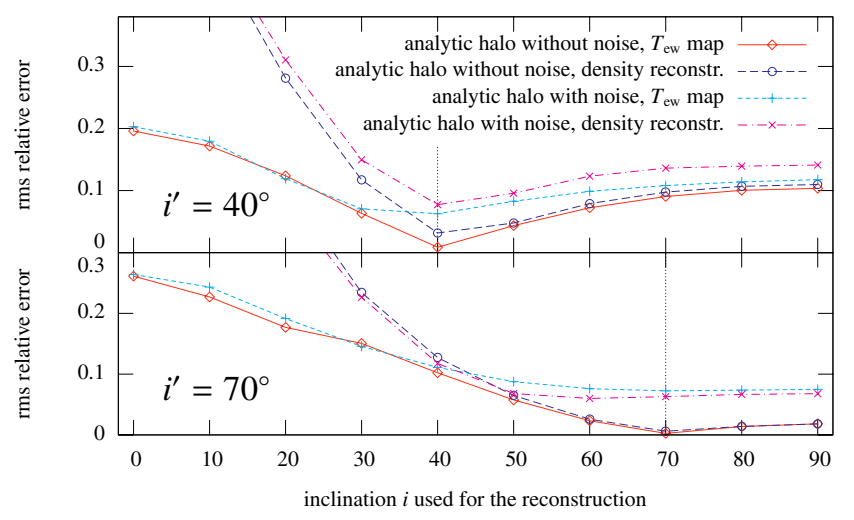

Fig. 12. Accuracy of emission-weighted temperature $T_{\text {ew }}$ maps and densities of reconstructed analytic halos. The deprojections started from maps obtained by projecting the analytic halo along a line-of-sight with inclination angles of $i^{\prime}=40^{\circ}$ and $i^{\prime}=70^{\circ}$. Inclination angles $i$ between $0^{\circ}$ and $90^{\circ}$ were used for the reconstruction. As expected, the best reconstructions are obtained for $i \approx i^{\prime}$. The errors are shown for deprojections from maps without observational noise and from smoothed maps with observational noise, and were averaged within a region of radius $500 h^{-1} \mathrm{kpc}$ around the map or halo centre.

$500 h^{-1} \mathrm{kpc}$ around the map centre and is shown for inclinations of $i^{\prime}=40^{\circ}$ and $i^{\prime}=70^{\circ}$ of the original halo. As desired, the minima of the error curves are at the correct locations $i \approx i^{\prime}$.

Note that the curves are only shown for $i$ between $0^{\circ}$ and $90^{\circ}$ because $\psi_{\mathrm{X} \text {-ray }}$ and $\psi_{\mathrm{SZ}}$ and hence the whole deprojection algorithm is insensitive to what is the front and what is the back side of the cluster. We thus get the same reconstruction and the same errors for deprojections which adopt inclination angles $i$ and $180^{\circ}-i$. There is no way to distinguish these cases from the $X$-ray, thermal Sunyaev-Zel'dovich effect and temperature maps. The error curves are thus symmetric about $i=90^{\circ}$. Also note that for the halo with noise, we added observational noise only to the X-ray flux maps and Sunyaev-Zel'dovich effect maps that were used for the reconstruction, but not to the $T_{\text {ew }}$ maps which we use for finding the inclination angle. We do not mimic observational noise in the temperature maps because it can only be realistically modeled when considering instrument response and line emission. In addition to the error curves of the temperature maps, we also show in Fig. 12 the volume-weighted, relative rms errors of the density reconstructions in the central $500 h^{-1} \mathrm{kpc}$. As expected, the reconstruction works best for $i \approx i^{\prime}$.

Figure 13 shows similar quantities as Fig. 12, but for the numerically simulated cluster halo g51. Original halo inclinations were set to $i^{\prime}=40^{\circ}$ and $i^{\prime}=68^{\circ}$, and $N_{\text {it }}=5$ iterations were used. The error curves are shown for the simulated halo without observational noise and using "radiusdependent smoothing", and including observational noise and using the complete smoothing scheme. No noise was added to the emission-weighted temperature maps. The relative rms $T_{\text {ew }}$ error was computed within a circle of radius $200 h^{-1} \mathrm{kpc}$ around the map centre, while the density reconstruction errors were again determined within the central $500 h^{-1} \mathrm{kpc}$. Also for this numerical halo the errors are smallest for $i \approx i^{\prime}$.

For halos with an original inclination $i^{\prime} \ll 90^{\circ}$ or $i^{\prime} \gg 90^{\circ}$ and for the analytic halos without noise, the minima of the error curves for the emission-weighted temperature and the density reconstructions are well defined. Thus the quality of the reconstruction of such halos depends strongly on using the correct inclination $i=i^{\prime}$ assumed in the deprojection. However, in such

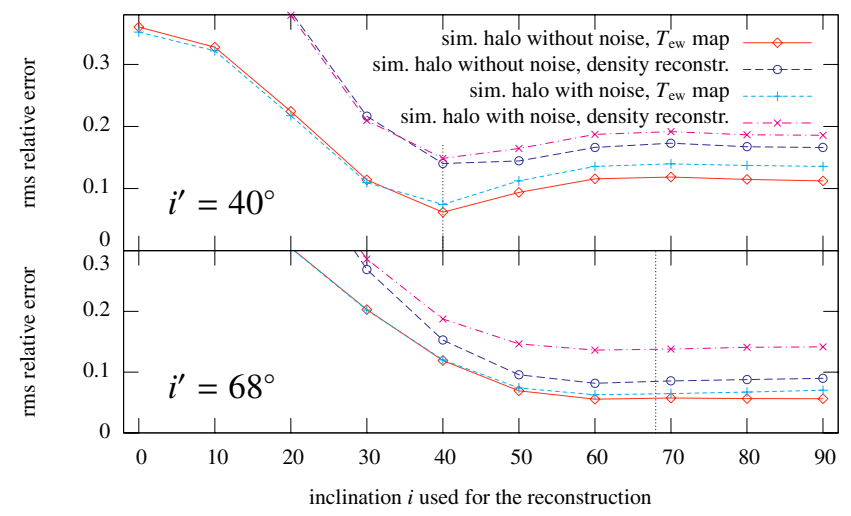

Fig. 13. Accuracy of emission weighted temperature $T_{\text {ew }}$ maps and densities for the reconstructed simulated halo g51. Maps obtained by projecting g51 along a line-of-sight with inclination angles of $i^{\prime}=40^{\circ}$ and $i^{\prime}=68^{\circ}$ were used. The reconstruction assumed inclination angles $i$ between $0^{\circ}$ and $90^{\circ}$. Best reconstructions are obtained for $i \approx i^{\prime}$. The errors are shown for deprojections from maps without observational noise but using "radius-dependent smoothing", and from maps with observational noise on which the complete smoothing scheme was applied. The rms relative errors were obtained within a circle of radius $200 h^{-1} \mathrm{kpc}$ around the map centre for the $T_{e w}$ maps and inside a sphere of radius $500 h^{-1} \mathrm{kpc}$ around the halo centre for the density reconstructions.

cases, the inclination angle is better constrained by the emissionweighted temperature maps. On the other hand, if the "symmetry" axis of the original halo is almost perpendicular to the lineof-sight, the minima of the error curves are usually broad, and finding the precise inclination $i=i^{\prime}$ for the reconstruction becomes less important. This can also be unterstood from the fact that deviations are symmetric around $i=90^{\circ}$. For example, the halo with an inclination of $i^{\prime}=68^{\circ}$ shown in Fig. 13 should exhibit minima at $i=i^{\prime}=68^{\circ}$ and at $i=180^{\circ}-i^{\prime}=112^{\circ}$ and a maximum in between. However, because these three extremal points are close to each other, they start merging into one broad minimum. Note that the emission-weighted temperature maps can constrain the inclination angle in both cases to values where the errors of the reconstruction are close to their minima.

The accuracy of inclination-angle estimates could be improved by using other independent information in addition to the temperature maps, such as data from weak and strong-lensing observations. Lensing observations allow reconstructions of the lensing potential (see e.g. Cacciato et al. 2005), which is simply the suitably rescaled projection of the lens' gravitational potential. A natural way to employ this for finding inclination angles is to assume hydrostatic equilibrium of the cluster gas in the potential well of the cluster and use the density and temperature reconstruction described above to obtain the gravitational potential of the reconstructed cluster halo. Its projection can then be compared to the lensing potential obtained from observations. Alternatively, one could use the deprojection algorithm discussed in Sect. 2.1 to obtain the three-dimensional gravitational potential from the lensing potential that was found from observations and compare it to the gravitational potential corresponding to the density and temperature reconstruction under the assumption of hydrostatic equilibrium. We shall explore these possibilities in a forthcoming study.

\section{Summary and discussion}

We propose a new method for deprojecting hot gas in galaxy clusters which combines X-ray and thermal Sunyaev-Zel'dovich 
effect observations and reconstructs three-dimensional density and temperature distributions. We start from the iterative deprojection algorithm suggested by Binney et al. (1990) which employs Richardson-Lucy deconvolution and assumes axial symmetry of the physical quantity whose three-dimensional distribution shall be reconstructed from two-dimensional maps of its projection along the line-of-sight.

This approach does not restrict the orientation of the symmetry axis to be parallel to the line-of-sight, but the inclination angle between the symmetry axis and the line-of-sight is assumed to be known. This algorithm runs into problems when it is used to reconstruct strongly peaked distributions such as the X-ray emissivity of a galaxy cluster. There, one obtains spike-shaped artifacts through the centre of the reconstructed halo. We suppress the formation of such artifacts by introducing a regularisation scheme for the iterative corrections used in the deprojection. Then, we generalise this algorithm to simultaneously reconstruct three-dimensional distributions of several physical quantities by combining two-dimensional maps of projections which probe these three-dimensional distributions in different ways.

Here, we discuss how three-dimensional density and temperature distributions of the intra-cluster medium can be reconstructed from combined X-ray and thermal Sunyaev-Zel'dovich effect observations. We test the method using synthetic data of analytically modeled and of numerically simulated galaxy clusters and discuss the quality of the reconstructions and the impact of observational noise, cluster substructure and deviations from axial symmetry. For numerical clusters which are of course not strictly axisymmetric, we use one of the principal inertial axes as the "symmetry" axis for the deprojection.

Our main findings, if we neglect observational noise and assume that the inclination angle between the symmetry axis and the line-of-sight is known, are:

- Spike-shaped artifacts of the deprojection are efficiently suppressed by the regularisation of the iterative corrections.

- Densities and temperatures of the ICM of axisymmetric analytic clusters can be reconstructed very accurately from X-ray flux and Sunyaev-Zel'dovich effect maps. Errors are of the order of $1 \%$ unless the angle between the symmetry axis and the line-of-sight is small.

- The three-dimensional density and temperature distributions of hot gas in numerically simulated clusters, although not strictly axisymmetric, can still be reliably reconstructed. Relative errors reach roughly $10 \%$. Smoothing of the X-ray flux and the Sunyaev-Zel'dovich effect maps can be used to suppress artifacts caused by subclumps.

- Accurate gas density and temperature profiles can be obtained from the reconstructions.

We then add photon noise corresponding to a total number of $10^{4}$ observed photons to the X-ray and observational noise corresponding to a four-hour ALMA Band 3 observation to the Sunyaev-Zel'dovich effect maps, respectively. We smooth the maps before repeating the deprojections to suppress small-scale fluctuations which the algorithm would otherwise attempt to approximate and thereby reduce the quality of the reconstructions. From the repeated reconstructions of the analytic and numerical halos, this time including observational noise, we conclude:

- Gas densities and temperatures of axisymmetric analytic halos can also be efficiently reconstructed from maps that contain observational noise. The relative errors of the reconstructions are about $5 \%$ to $10 \%$.

- The three-dimensional structure of the ICM of numerically simulated clusters can also be reliably reconstructed from $\mathrm{X}$-ray flux and Sunyaev-Zel'dovich effect maps that contain observational noise. Relative errors reach roughly $15 \%$.

- Accurate profiles can be obtained from the reconstructions.

- Five iterations are sufficient for the ICM deprojection. Using a larger number does not increase the quality of the reconstruction.

For these deprojections, we assumed that the inclination angle between the symmetry axis and the line-of-sight is known beforehand. This will usually not be the case for observations of real clusters. In principle, one could try to find the inclination angle by deprojecting the cluster assuming different values of the inclination angle and comparing the original X-ray and Sunyaev-Zel'dovich effect maps to those expected from the reconstructed halo. They should match best if the correct inclination is used for the deprojection. Unfortunately, the minima of the deviations of the maps as a function of the assumed inclination angle are quite broad and not always centred on the correct value. However additional data which are independent of the $\mathrm{X}$-ray flux and the Sunyaev-Zel'dovich effect maps, can provide information on the inclination angle. We show that high-quality emission-weighted temperature maps which become more and more routinely available, can constrain the inclination angle of a cluster's symmetry axis to values for which the quality of the reconstruction is close to its optimum.

Acknowledgements. We are deeply indebted to Klaus Dolag, who generously provided us access to the numerical simulations of the cluster sample that was used in this work. We also thank Massimo Meneghetti for useful discussions. E.P. is supported by the German Science Foundation under grant number BA 1369/6-1.

\section{References}

Arnaud, K. A. 1996, Astronomical Data Analysis Software and Systems V, ASP Conf. Ser., 101, 17

Binney, J. J., Davies, R. L., \& Illingworth, G. D. 1990, ApJ, 361, 78

Butler, B., \& Wootten, A. 1999, ALMA Memo, 276,

http://www.alma.nrao.edu/memos/html-memos/

abstracts/abs276.html

Cacciato, M., Bartelmann, M., Meneghetti, M., \& Moscardini, L. 2005, A\&A, submitted, preprint [arXiv: astro-ph/0511694]

Cavaliere, A., \& Fusco-Femiano, R. 1976, A\&A, 49, 137

De Filippis, E., Sereno, M., Bautz, M. W., \& Longo, G. 2005, ApJ, 625, 108

Dolag, K., Vazza, F., Brunetti, G., \& Tormen, G. 2005, MNRAS, 364, 753

Doré, O., Bouchet, F. R., Mellier, Y., \& Teyssier, R. 2001, A\&A, 375, 14

Jing, Y. P., \& Suto, Y. 2002, ApJ, 574, 538

Lee, J., \& Suto, Y. 2004, ApJ, 601, 599

Lucy, L. B. 1974, AJ, 79, 745

Lucy, L. B. 1994, A\&A, 289, 983

Mazzotta, P., Rasia, E., Moscardini, L., \& Tormen, G. 2004, MNRAS, 354, 10

Puchwein, E., Bartelmann, M., Dolag, K., \& Meneghetti, M. 2005, A\&A, 442, 405

Reblinsky, K. 2000, A\&A, 364, 377

Reblinsky, K., \& Bartelmann, M. 2001, in Gravitational Lensing: Recent Progress and Future Goals, ASP Conf. Ser., 237, 337

Springel, V. 2005, MNRAS, 364, 1105

Springel, V., Yoshida, N., \& White, S. D. M. 2001, New Astron., 6, 79

Tormen, G. 1997, MNRAS, 290, 411

Yoshida, N., Sheth, R. K., \& Diaferio, A. 2001, MNRAS, 328, 669

Zaroubi, S., Squires, G., de Gasperis, G., et al. 2001, ApJ, 561, 600

Zaroubi, S., Squires, G., Hoffman, Y., \& Silk, J. 1998, ApJ, 500, L87 